\title{
Neue Erkenntnisse zur Plasmamodifizierung von Polymeroberflächen
}

\author{
Dr. rer. nat. habil.Jörg Friedrich, Dr. rer. nat. Dipl.-Chem. Lothar Wigant, Dr. rer. nat. Wolfgang Unger, \\ Dipl.-Ing. Andreas Lippitz, Dipl.-Chem. Sh. Geng, Dipl.-Chem. J. Erdmann, Dipl.-Ing. H.-V. Gorsler
}

\section{Zum Plasmaprozeß}

Zweck der Plasmabehandlung

Die Benetz-, Verkleb- und Metallisierung von Polymeren mit niedriger Oberflächenenergie kann mit genügend hoher Geschwindigkeit nur in elektrischen Entladungen erreicht werden. Verweilzeiten von etwa 0,01 bis $10 \mathrm{~s}$ in oxidierenden Plasmen elektrischer Entladungen sind dazu notwendig. Auch die Effektivität der Modifizierung in einer elektrischen Entladung übertrifft in der Regel die Wirkung chemischer oder physikalischer Verfahren.

\section{Niederdruckplasma}

Besonders bewährt hat sich der Einsatz von NiederdruckGlimmentladungen, die mit Gleichstrom, Hochfrequenzoder Mikrowellenenergie erzeugt werden können. Derartige Niederdruckplasmen sind von der Nichtisothermie der einzelnen Plasmabestandteile geprägt, d. h. zwischen den Plasma-Subsystemen der schweren Teilchen (Atome, Moleküle, Ionen) auf der einen Seite und dem

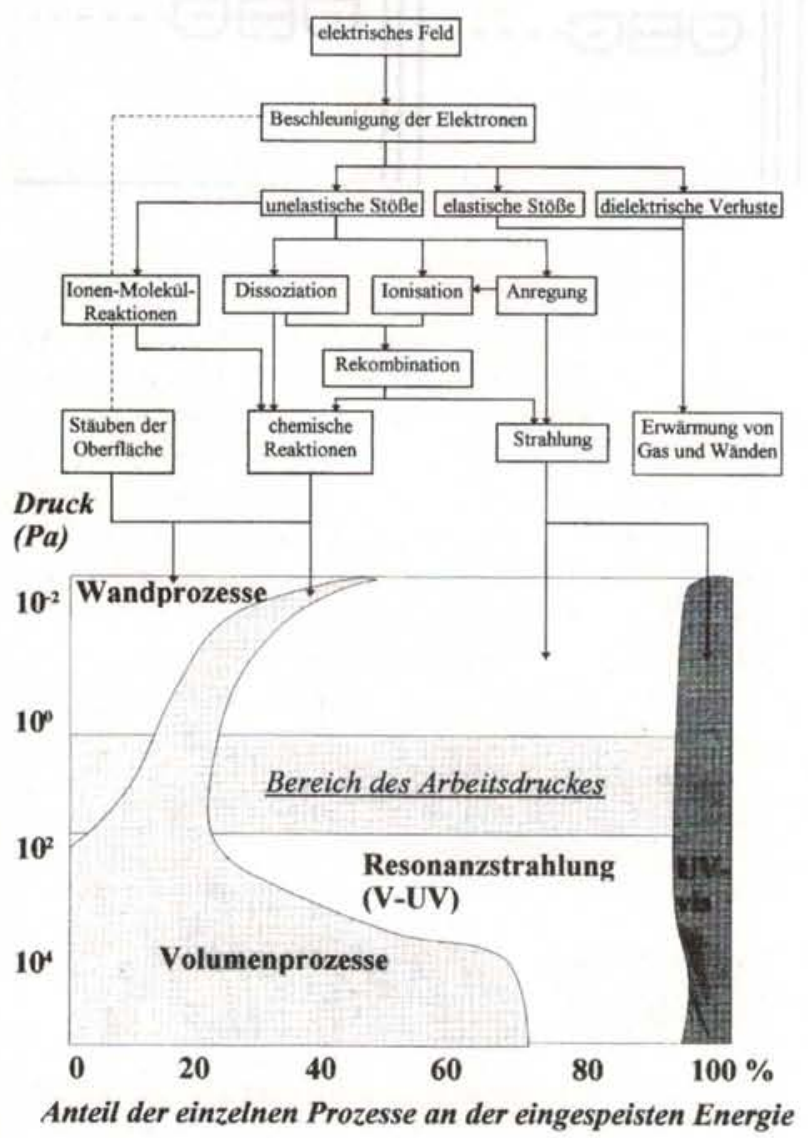

Abb. 1: Verteilung der elektrischen Energie in einer Niederdruckentladung auf einzelne Elementarprozesse sowie ungefähre prozentuale Verteilung der eingekoppelten elektrischen Energie auf die wichtigsten Plasmaprozesse
Subsystem der Elektronen auf der anderen Seite besteht ein energetisches Ungleichgewicht [1]. Im einzelnen bedeutet das eine relativ hohe kinetische Energie bzw. wegen $\mathrm{m} / 2 \mathrm{v}^{2}=3 / 2 \mathrm{kT}$ eine hohe Temperatur für die Elektronen und eine niedrige Temperatur des Neutralgases sowie der lonen. Ursache für diese Erscheinung ist der große Masseunterschied zwischen Elektronen und schweren Teilchen. Wegen des Impuls- und des Energieerhaltungssatzes können die Elektronen ihre im elektrischen Feld ërworbene kinetische Energie nicht durch elastische Stöße an die schweren Teilchen oder die Wand abgeben. Es baut sich eine Maxwell-Boltzmann-Energieverteilung der Elektronen auf mit einer durchschnittlichen Energie von einigen eV [2]. Die Elektronen des „hochenergetischen Schwanzes" der Energieverteilung überschreiten die Anregungs- bzw. Ionisationsenergie der Atome und Moleküle, was zu den sogenannten unelastischen Stößen der Elektronen führt. In Abb. 1 sind die verschiedenen Energieumwandlungsprozesse im nichtisothermen Plasma dargestellt. Zusätzlich ist im unteren Teil der Abbildung die ungefähre prozentuale Verteilung der Energie auf die einzelnen Elementarprozesse angegeben. Die in Abb. 2 wiedergegebene ElektronenEnergieverteilungsfunktion zeigt die Diskriminierungen im Bereich hoher Energie (etwa $>3 \mathrm{eV}$ ), wo die Anregung und lonisation des Plasmagases einsetzt [2]. Auf die organische Polymeroberfläche bezogen heißt das in der Regel, daß die auftreffenden Teilchen oder Photonen mindestens 3,5 eV Energie liefern müssen, um eine chemische Reaktion in Gang zu setzen. Diese 3,5 eV sind notwendig, um die in Polymeren am häufigsten anzutreffenden C-C- und C-H-Bindungen spalten zu können [3]. Stabile organische Moleküle oder Polymerbausteine, wie aromatische Ringe, benötigen $>6 \mathrm{eV}$, um C-C-Bindungen zu dissoziieren. Einige wichtige elementare Reaktionen im Plasma und an den Wänden und bei der Wechselwirkung Plasma-Polymeroberfläche sind in Abb. 3 aufgelistet. Allein die Zahl der aufgefuihrten Elementarund Folgereaktionen im Plasmavolumen, in der Grenzschicht Plasma-Polymer und in oberflächennahen Schichten des Polymers macht auf die im chemischen Sinne häufig fehlende Selektivität der Plasmaprozesse aufmerksam, d. h. die Behandlung im Plasma führt in den meisten Fällen zu einem großen Spektrum an Reaktionsprodukten. Dieser offensichtliche Nachteil vieler plasmachemischer Prozesse wird jedoch bei der Vorbehandlung von Polymeroberflächen für die Verklebung oder Metallisierung dadurch kompensiert, daß praktisch alle Arten plasmaerzeugter funktioneller Gruppen zur Adhäsion beitragen (Dipol-Dipol-Wechselwirkungen, chemische Bindungen). 


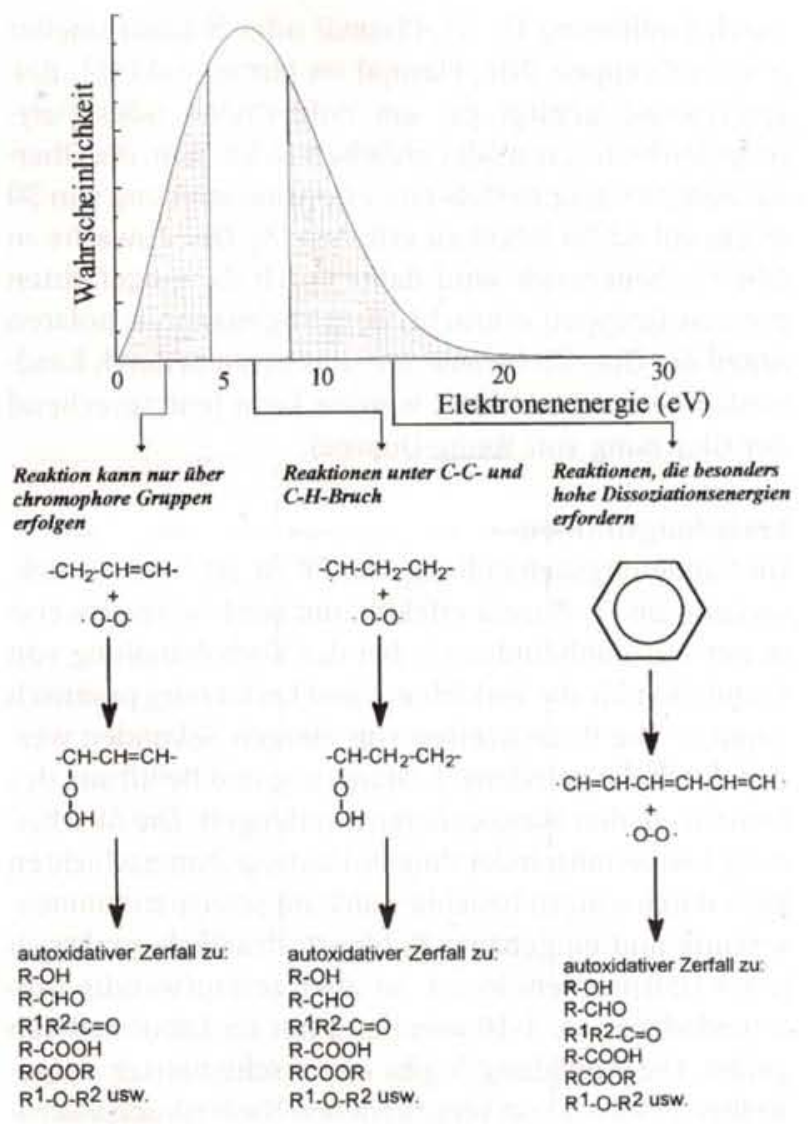

Abb. 2: Verteilung der Elektronenenergie in einer elektrischen Niederdruckentladung und Zuordnung einzelner chemischer Reaktionsgruppen zu bestimmten Elektronenenergiebereichen

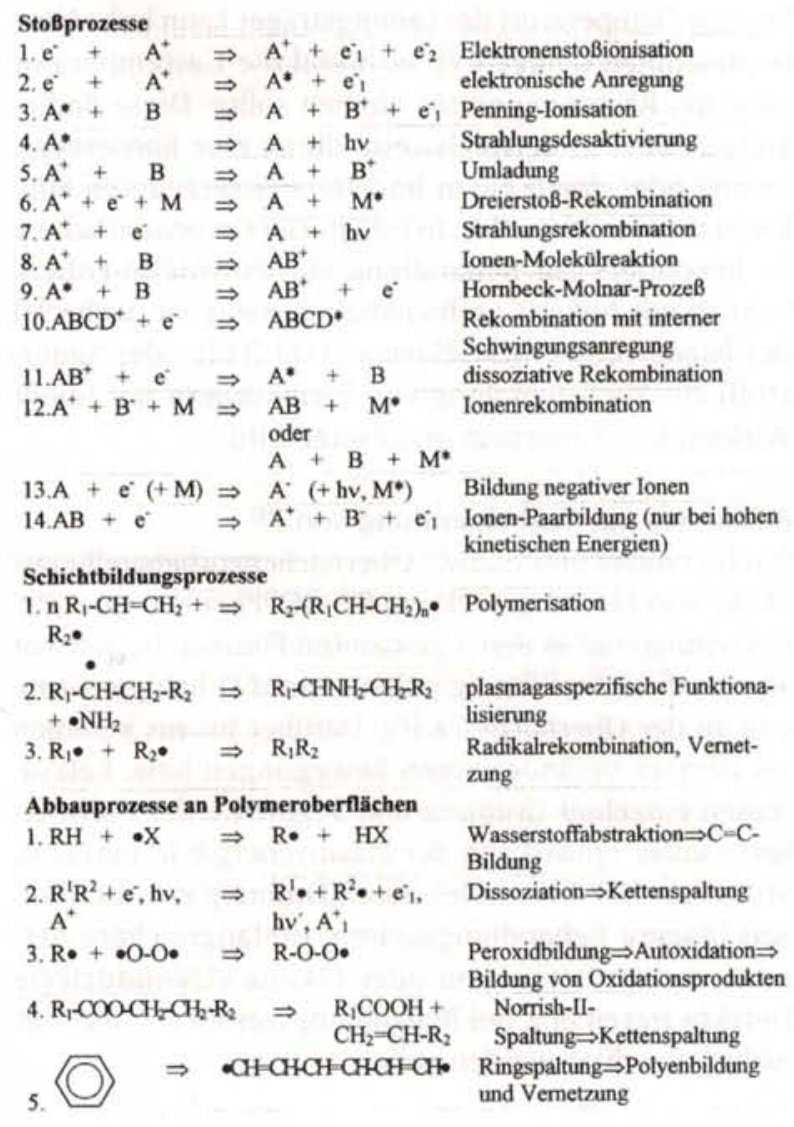

Abb. 3: Verkürzte Übersicht über elementare Stoßprozesse im nichtisothermen Plasma, Polymerschichtbildungsreaktionen und Abbauprozesse von Poly. meren in oxidierenden Plasmen
Rolle der Polymerstruktur und -zusammensetzung Das Polymer und sein Oberflächenzustand sind ebenfalls von komplexer und komplizierter chemischer und physikalischer Struktur geprägt. Apolare Polyolefine, wie Polyethylen oder Polypropylen, besitzen eine komplizierte morphologische Struktur, die von amorphen geknäuelten Strukturen bis hochorientierten kristallinen Strukturen geprägt ist, die wiederum in charakteristischen Überstrukturen angeordnet sind (z. B. Sphärolithe, Dendrite). Handelt es sich um Polymerfolien oder Fasern, dann tritt noch eine durch Reckung hervorgerufene charakteristische mono-, bi- oder uniaxiale Orientierung der Molekülketten hinzu. In Abb. 4 ist beispielsweise ein Ausschnitt aus der Struktur eines teilkristallinen Polyethylens gezeigt (ohne Reckorientierung) und der Ort des bevorzugten Angriffs des Plasmas dargestellt (hier: Anlagerung $\mathrm{O}$-funktioneller Gruppen im $\mathrm{O}_{2}$-Plasma).

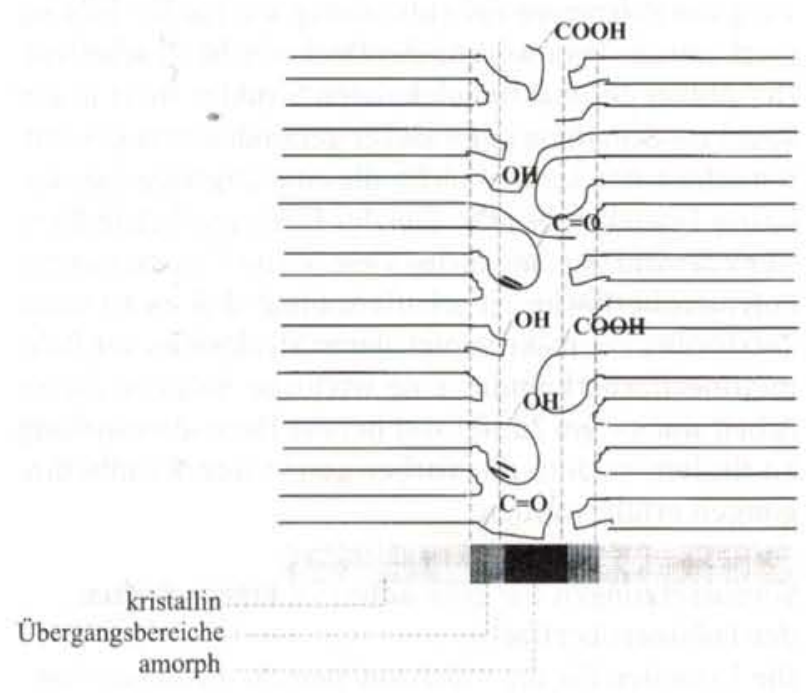

Abb. 4: Modell einer Grenzfläche zwischen kristallinen und amorphen Bereichen in Polyethylen und Lokalisierung der bevorzugten Anlagerung von funktionellen Gruppen an das Polymer (Sauerstoffplasma)

\section{Tiefenwirkung des Plasmas}

Die Plasmamodifizierung von Polymeren ist nicht auf die unmittelbare Oberfläche beschränkt. Während das Plasmapartikelbombardement tatsächlich nur wenige Nanometer Eindringtiefe besitzt, kann insbesondere bei längeren Behandlungszeiten ( $>10 \mathrm{~s}$ ) die Plasma-VakuumUV-Strahlung Strahlungsschäden unterschiedlicher Art und in z. T. beträchtlicher Entfernung von der Oberfläche anrichten (Mikrometerbereich). Typische Strahlenschäden sind: Radikalbildung, Anlagerung von Luftsauerstoff an abgeschirmte Radikale im Polymervolumen, Vernetzung, Bildung von Doppelbindungen durch $\mathrm{H}$-Abstraktion, Veränderung der Orientierung anisotroper Strukturen und Verlust an übermolekularer Struktur [4].

\section{Zusammenhang zwischen Plasmabeständigkeit und Polymerstruktur}

Die unterschiedlichen chemischen Grundstrukturen der Polymere besitzen einen erheblichen Einfluß auf die Ätzbeständigkeit, beispielsweise gegenüber einem Sauerstoffplasma. Sauerstoffenthaltende Polymere sind häufig leichter abbaubar, aromatische Strukturen und Vernetzungen können stabilisierend wirken, Seiten- 
gruppen werden bevorzugt angegriffen, ebenso Säure-, Peroxi- oder Carbonatstrukturen, in denen Abbauprodukte schon vorgeformt sind $\left(\mathrm{CO}, \mathrm{CO}_{2}\right)$, um nur einige wenige allgemeine Linien zu schildern [5]. Das Verhalten der Polymere gegenüber einem Sauerstoffplasma ähnelt dem Verhalten gegenüber ionisierender und UV-Strahlung (Photooxidation), wird aber auch durch Spezifika geprägt, wie Angriff und Zerstörung aromatischer Strukturen und vergleichsweise sehr hohe Ätzraten.

\section{Zeitlicher Ablauf der Reaktionen}

Zur optimalen Haftvorbereitung von Polymeren fuir die Verklebung oder Metallisierung ist die Kenntnis vom zeitlichen Ablauf der chemischen Reaktionen an der Polymeroberfläche sehr wichtig, weil unbedingt der Abbau des Polymers, d. h. die Spaltung der Makromolekuilketten vermieden werden sollte (Ausnahme: Anätzung von Polymeren zur Aufrauhung, um Punkte für eine mechanische Verankerung der Deckschicht zu schaffen). Der Abbau der makromolekularen Struktur fuihrt in der Regel zur Schaffung einer locker gebundenen sogenannten schwachen Grenzschicht, die eine ungeniigende kohäsive Festigkeit besitzt. Ziel der Plasmavorbehandlung ist es demzufolge, möglichst viele polare Gruppen an der Polymeroberfläche zu schaffen, ohne daß es zu einer Zerstörung der makromolekularen Struktur an der Polymeroberfläche kommt. Eine wichtige Aufgabe dieser Arbeit war es, ein Zeitfenster bei der Plasmabehandlung zu finden, in dem die vorher genannten Randbedingungen erfuillt werden.

\section{Voraussetzungen für gute adhäsive Eigenschaften der Polymeroberfläche}

Die Ursachen für die Adhäsion zwischen Polymer-Polymer (z. B. einem Kleber) oder Polymer-Metall sind thermodynamische (z. B. Angleichung der Oberflächenenergien von Polymer und Deckschichtmaterial), elektrostatische (Bildung einer elektrischen Doppelschicht), chemische Effekte (Bildung grenzflächenübergreifender chemischer Bindungen zwischen Polymer und Deckschicht), mechanische Parameter (Erzeugung von Rauhigkeit und damit Verhakung von Polymer und Deckschicht an der Grenzfläche, ,Druckknopfmechanismus“) oder Interdiffusion (partielle Durchdringung der Makromolekülketten von Polymer und Kleber an der Grenzfläche) [7]. Bei der Verklebung oder Metallisierung von Polyolefinen oder anderen Polymeren mit niedriger Oberflächenenergie steht die Erhöhung der Oberflächenenergie

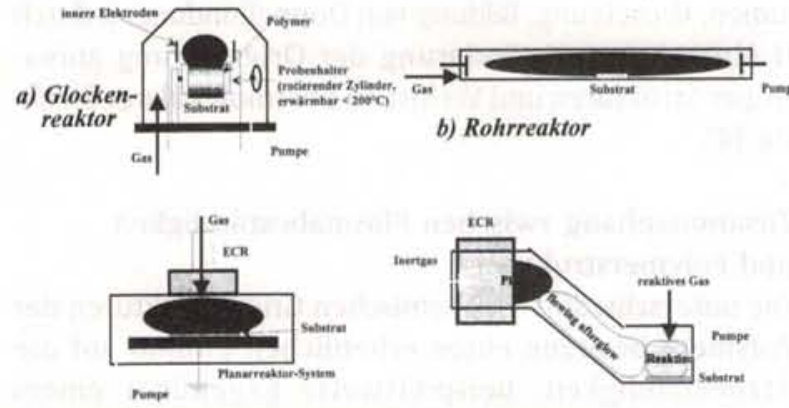

c) Elektron-Zyklotron-Resonanz-Plasma d) Down-stream-Plasma

$A b b .5$ : Schematische Gegenüberstellung von verschiedenen Ausführungsmöglichkeiten von Niederdruck-Plasmareaktoren durch Einfuihrung $\mathrm{O}-\left(\mathrm{O}_{2}\right.$-Plasma) oder $\mathrm{N}$-funktioneller polarer Gruppen ( $\mathrm{NH}_{3}$-Plasma) im Mittelpunkt [6]. Beispielsweise genügt es, um Polyethylen oder Polypropylen bedrucken oder anfärben zu können, die Oberflächenspannung mittels einer Coronaentladung von 30 $\mathrm{mN} / \mathrm{m}$ auf $42-50 \mathrm{mN} / \mathrm{m}$ zu erhöhen [8]. Der Zuwachs an Oberflächenenergie wird dabei durch die eingefärbten polaren Gruppen erbracht, dem sogenannten polaren Anteil der Oberflächenenergie, was bequem durch Randwinkelmessung ermittelt werden kann (entrsprechend der Gleichung von Young-Dupreé).

\section{Entladungsformen}

Die Entladungsbehandlung von PP ist als Niederdruckvariante im $\mathrm{O}_{2}$-Plasma effektiv und wird beispielsweise in der Automobilindustrie bei der Vorbehandlung von Formteilen für die Verklebung und Lackierung praktisch genutzt. Die Prozeßzeiten von einigen Sekunden werden durch die Beladung, Evakuierung und Belüftung des Reaktors in den Minutenbereich verlängert. Die Abscheidung haftvermittelnder duinner Plasmapolymerschichten kann durch nahezu beliebig wählbare Schichtzusammensetzung und eingebaute Kohlenstoffradikale praktisch jedes Haftproblem lösen, ist aber zeitaufwendig (Abscheidedauer ca. 1-10 min.) und nur im Labormaßstab gelöst. Die Abbildung 5 gibt einen schematisch dargestellten Eindruck von verschiedenen Niederdruckplasmareaktortypen wieder.

Anwendungstechnisch bevorzugte Plasmabehandlungen bei Normaldruck, z. B. an Luft, sind sehr schnell (ca. 0,01$1 \mathrm{~s})$. Auch die Entladungen bei Normaldruck sollten vorzugsweise nichtisothermen Charakter haben, d. h. die Energie (Temperatur) der Ladungsträger kann hohe Werte annehmen (einige $\mathrm{eV}$ ), während die Gastemperatur nahe bei Raumtemperatur bleiben sollte. Diese Bedingungen werden beispielsweise durch eine Barriereentladung oder durch einen hochfrequenzerzeugten Funkenplasmastrahl (s. Abb. 6) erfuillt. Die Coronaentladung ist besonders zur Behandlung von Polyolefin-Folienbahnen mit hoher Geschwindigkeit geeignet, während der Niedertemperatur-Plasmastrahl (Luft oder Sauerstoff) zur Vorbehandlung von Formkörpern mit hoher Wirksamkeit bevorzugt eingesetzt wird.

\section{Bekanntes zur Vorbehandlung von PP}

Der hier näher untersuchte Oberflächenvorbehandlungseffekt von biaxial gerecktem PP (BOPP)-Folien oder PPPlattenmaterial in den 3 genannten Plasmen basiert vor allem auf der Einfuihrung polarer, meist O-haltiger Gruppen an der Oberfläche $[9,10]$. Darüber hinaus kommen als weitere Veränderungen Bewegungen bzw. Relaxationen einzelner Gruppen und Segmente der Polymerkette unter Einwirkung der Plasmaenergie in Betracht. Störungen der übermolekularen Ordnung erfordern etwas längere Behandlungszeiten. Umfangreichere Ätzprozesse, Vernetzungen oder Plasma-VUV-induzierte Defekte treten erst bei Behandlungszeiten auf, die hier nicht betrachtet werden sollen.

\section{Aufgabenstellung}

In dieser Arbeit werden neue Erkenntnisse zur Oberflächenmodifizierung von Polyolefinen im Plasma darge- 


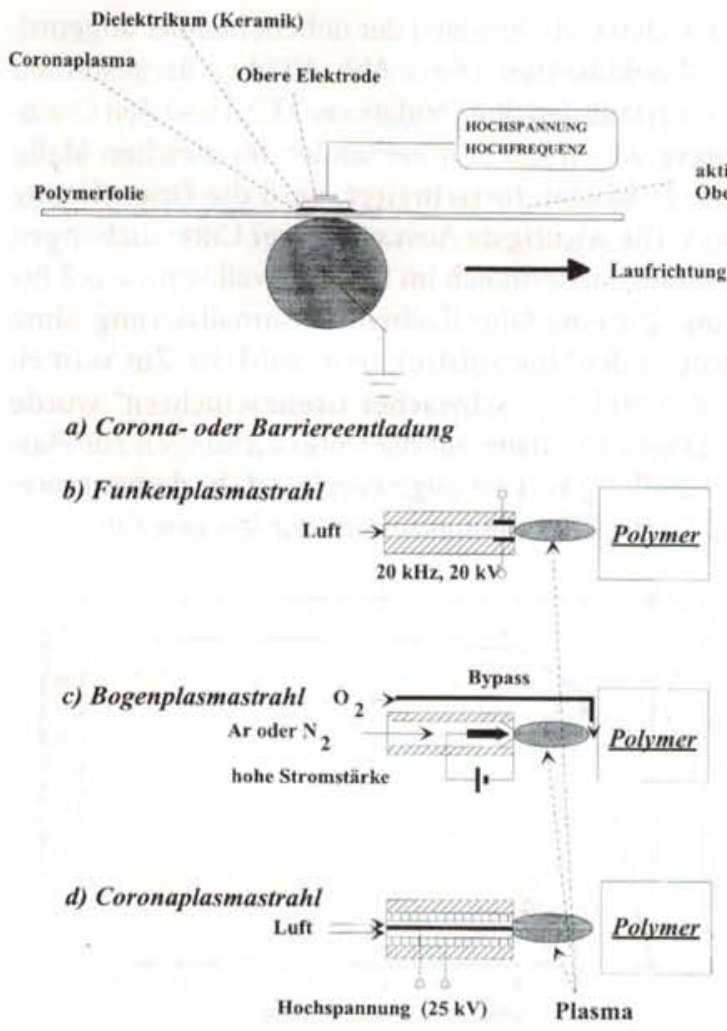

Abb. 6: Verwendete Typen von Normaldruck-Plasmageräten

stellt, die durch Nutzung moderner Oberflächenanalytik erhalten wurden. Diese oberflächensensitiven Methoden gestatteten die Verwendung von hochgeordneten monomolekularen $\mathrm{C}_{18}$-Aliphatenschichten als Modell für eine Polyolefineoberfläche. Mittels dieser Modelle konnten Plasmaeinwirkungen bei Kurzzeitbehandlungen $(0,1$ 10 s) hinsichtlich Chemismus und molekularer Umstrukturierung an Aliphatenoberflächen untersucht werden. Ein zweites Anliegen dieser Arbeit war der Vergleich der Effektivität der o. g. Plasmaverfahren bei der PolymerOberflächenaktivierung.

\section{Geräte und Methoden}

Zur Normaldruck-Plasmabehandlung des Polypropylens wurden eine Coronaentladungsanlage und ein Funkenplasmastrahlgerät (3D-Treater) der Fa. AHLBRANDT benutzt. Die Niederdruck-Glimmentladung wurde als Gleichstrom- oder Hochfrequenzplasma in einem evakuierten Rezipienten (LEYBOLD) erzeugt. Als Plasmagase kamen Luft, bzw. Luft-Sauerstoff-Gemische (1:1) oder reiner Sauerstoff zum Einsatz.

Die Prüfung der Veränderung der Oberflächenbenetzbarkeit erfolgte durch automatisierte Randwinkelmessung (KRÜSS G1/G40) nach der Methode von Fowkes, Owen, Wendt und Rabel mit mehreren Testflüssigkeiten. Die chemischen Veränderungen an den PP-Oberflächen wurden durch XPS (ESCA), NEXAFS (Near Edge X-ray Absorption Fine Structure), SEIRA (Surface Enhanced FTIR Absorption)-Spektroskopie, MALDI-TOF (Matrix Assisted Laser Desorption Ionization-Time of Flight Mass Spectrometry) und anderen Methoden ermittelt. XPS (Röntgen-Photoelektronenspektroskopie) wurde zur Ermittlung der Elementzusammensetzung und zur Bestim- mung chemischer Strukturelemente an der Oberfläche benutzt. NEXAFS ist eine neue Methode, die Aussagen zur räumlichen Ausrichtung von C-C-, C-H, C=C-, C-Ound $\mathrm{C}=\mathrm{O}$-Strukturen liefert. Dazu wird Synchrotronstrahlung in verschiedenen Winkeln zur Oberfläche eingestrahlt und der senkrecht dazu stehende E-Vektor der Strahlung tritt bei anisotrop aufgebauten Schichten mit entprechend orientierten Bindungsorbitalen in Wechselwirkung. Verschiedene elektronische Übergänge in den Schichtmolekülen werden angeregt und resultierende Photoelektronen oder Fluoreszenzstrahlung werden gemessen. SEIRA benutzt insuläre metallische Dünnstschichten (ca. $3 \mathrm{~nm})$, an denen Schichten adsorbiert werden, wobei durch Mehrfachreflexion monomolekulare Schichten analysiert werden können. MALDI nutzt die unzerstörte matrixunterstützte Desorption von oligomeren und polymeren Molekülen durch Lasereinstrahlung zur Molekulargewichtsbestimmung durch ein Flugzeitmassenspektrometer im Bereich von 500 bis maximal 500000 Dalton.

Mit PP-Plattenmaterial wurden überlappte Verklebungen mit einem feuchtehärtenden PU-Kleber (BETAMATE 7020, Gurit-Essex AG) vorgenommen, um die Hafteigenschaften des Polymers nach der jeweiligen Plasmavorbehandlung prüfen zu können. Die Prüflinge wurden vor der Zugschermessung einer intensiven Bewitterung ausgesetzt.

\section{Ergebnisse}

\section{Modellschichten}

Die hochorientierten aliphatischen Modellschichten (prinzipielle Struktur s. Abb. 7) wurden in einem Sauerstoff-Niederdruckplasma behandelt (Abb. 7). Der zeitliche Verlauf zeigt sich im XPS-C1s-Spektrum als Verbreiterung des Peaks zu höheren Bindungsenergien, was durch funktionelle Gruppen der Typen $\mathrm{C}-\mathrm{O}-, \mathrm{C}=\mathrm{O}$ und $-\mathrm{O}-\mathrm{C}=\mathrm{O}$ verursacht wird. Die $\mathrm{C}_{18}$-Modellschicht erreicht bereits nach $4 \mathrm{~s}_{2}$-Plasmabehandlung den maximalen $\mathrm{O}$ Gehalt von ca. 30 at\% O (Abb. 7). Längerdauernde Behandlungen fuihren ausschließlich zum Abbau der Schicht. Diese Aussagen könnten durch SEIRA-Messungen bestätigt werden (Abb. 8). Nach einer $10 \mathrm{~s}$ Behandlung löst sich die Schicht vom Substrat (geringere Intensität der Carboxylatbande), die Oxidation ist weit vorangeschritten (intensive Carbonylbande) und die aliphatische Kette

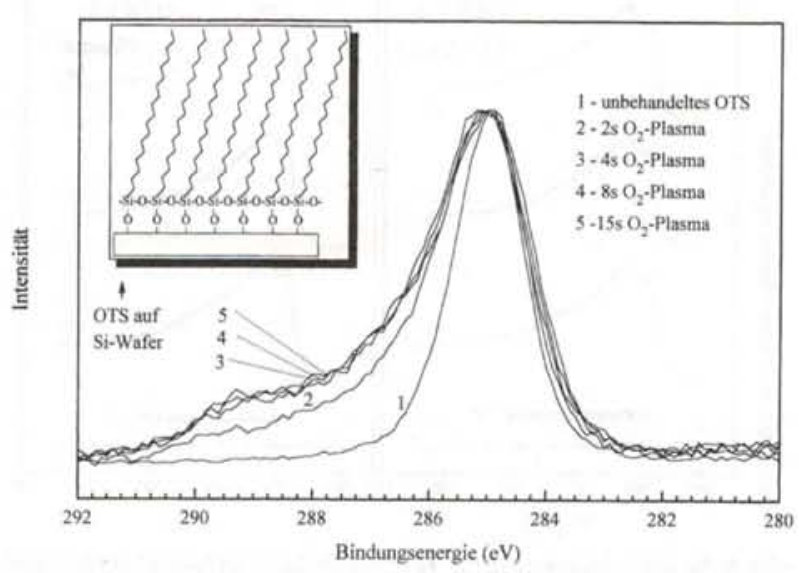

Abb. 7: Cls-Peak einer $\mathrm{C}_{18} \mathrm{H}_{37}$-Modellschicht in Abhängigkeit von der Behandlungszeit im $\mathrm{O}_{2}$-Plasma 


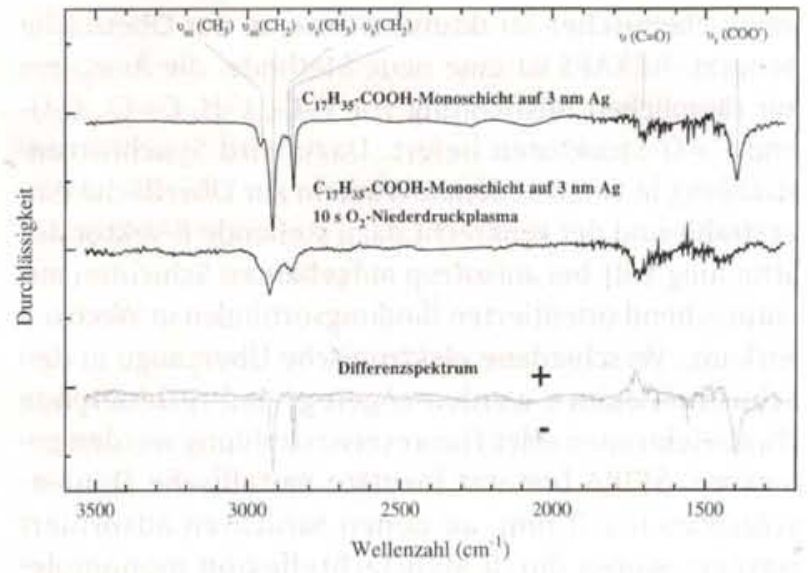

Abb. 8: SEIRA-Spektrum einer $\mathrm{C}_{1} \mathrm{H}_{35}$-Modellschicht (Stearinsäure) vor und nach 02-Plasmabehandlung auf einer $3 \mathrm{~nm}$ dicken Silberschicht (inselförmige. Ag-Cluster)

ist teilweise abgebaut $\left(\mathrm{CH}_{\mathrm{x}}\right.$-Valenzschwingungen bei 299 $\mathrm{cm}^{-1}$ besitzen geringere Intensität und sind verbreitert). Die NEXAFS-Messungen dieser aliphatischen Modellschichten geben über molekulare Umstrukturierungen Auskunft. Die bei 3 verschiedenen Einstrahlwinkeln der Synchrotronstrahlung $\left(20,55,90^{\circ}\right)$ aufgenommenen Spektren sind in Abb. 9 dargestellt. Aus der Differenz zwischen $20^{\circ}$ - und $90^{\circ}$-Spektren kann eine Ableitung der Orientierung der Molekülketten vorgenommen werden. Wegen des $25^{\circ}$-Kippwinkels der Moleküle zur Senkrechten auf der Substratoberfläche kann der E-Vektor der Synchrotronstrahlung $\left(90^{\circ}\right.$ zur Strahlrichtung) bei $20^{\circ}$ Einstrahlwinkel (bezogen auf die Oberfläche) mit allen C-C-Orbitalen der Kette wechselwirken, bei $90^{\circ}$ vorwie-
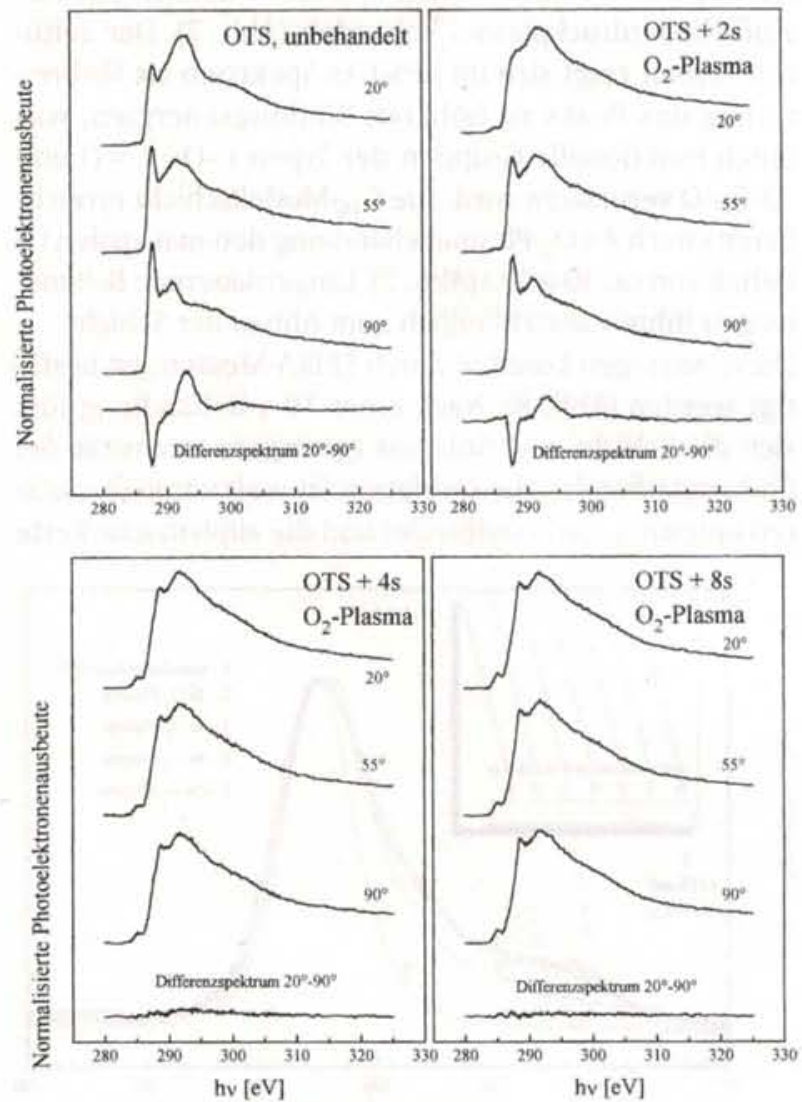

Abb. 9: NEXAFS-Spektren einer $\mathrm{C}_{18} \mathrm{H}_{37}$-Modellschicht (Octadecyltrichlorsilan) auf Si-Wafern nach unterschiedlich langen Behandlungszeiten im $\mathrm{O}_{2}$-Niederdruckplasma gend mit den C-H-Orbitalen der nebeneinander angeordneten Molekülketten. Die in Abb. 10 oben dargestellten Kurven verknüpfen den Oxidations- (XPS) und den Orientierungsgrad (NEXAFS) miteinander. Im gleichen Maße wie die Oxidation fortschreitet, wird die Orientierung zerstört. Die wichtigste Aussage dieser Untersuchungen ist nun die, daß lediglich im Zeitintervall von ca. 0,2 bis maximal $2 \mathrm{~s}$ eine Oberflächenfunktionalisierung ohne Zerstörung der Molekülstruktur möglich ist. Zur Vermeidung der Bildung „Schwacher Grenzschichten“ wurde diese Erkenntnis dann auf die Untersuchungen zur Plasmabehandlung von PP angewendet, d. h. dementsprechend kurze Behandlungszeiten wurden gewählt.
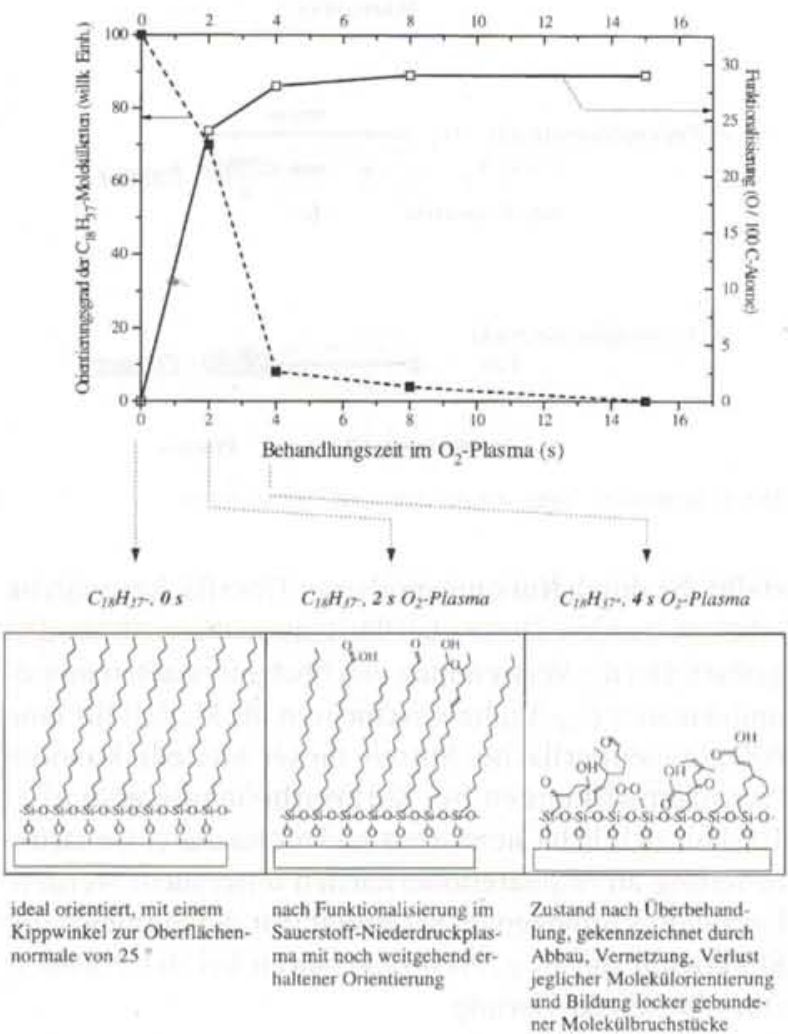

Abb. 10: Zusammenhang zwischen Ordnungsgrad und 0-Funktionalisierung einer $\mathrm{C}_{15} \mathrm{H}_{37}$-Modellschicht sowie Interpretation

\section{PP-Oberfläche}

Die NEXAFS-Spektren von BOPP weisen auf eine geringe Orientierung der Molekülketten hin und zeigen nach $\mathrm{O}_{2}$-Plasmabehandlung deutliche Veränderungen (Abb. 11). Die Kettenausrichtung (Orientierung) sinkt mit der Behandlungszeit, die Zahl der schon im Ausgangszustand vorhandenen $\mathrm{C}=\mathrm{C}$-Bindungen in der einige Nanometer dicken analysierten Oberflächenschicht wird so stark erhöht, daß konjugierte Systeme entstehen (oberes Spektrum), und der Einbau von C-O- und C $=\mathrm{O}$-Strukturen wird deutlich erkennbar (unteres Spektrum).

Die Funktionalisierung der PP-Oberfläche mit O-haltigen Gruppen im $\mathrm{O}_{2}$-Niederdruckplasma entspricht der der Modellschichten (s. Abb. 7), während im Funkenplasmastrahl ( $\left.\mathrm{Luft} / \mathrm{O}_{2}\right)$ und in der Coronaentladung ein etwa 10 mal schnellerer Ablauf erreicht wird. Die Oberflächenoxidation von PP in der Coronaentladung ist im Vergleich zu den anderen Plasmabehandlungen so stark, daß sie mittels FTIR-ATR-Technik nachgewiesen werden kann (Abb. 12). 

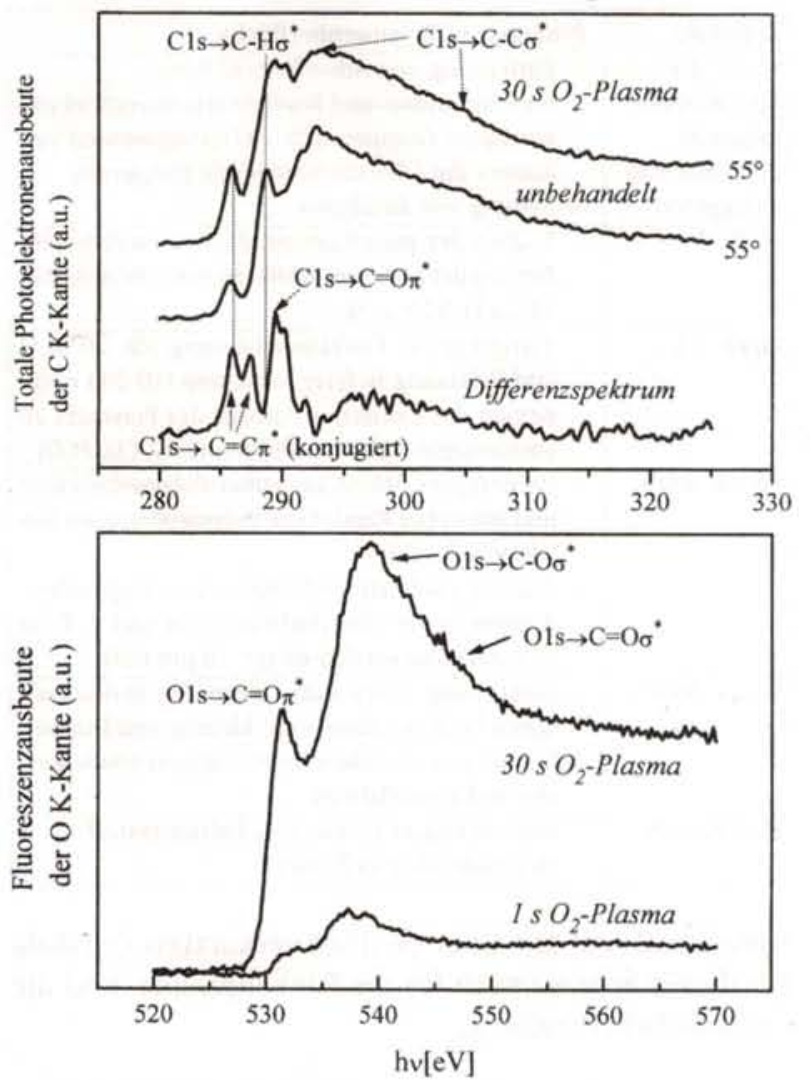

Abb. 11: NEXAFS-Spektren von O2-Plasma-behandeltem PP (oben C-K-Kante, unten 0 -K-Kante)

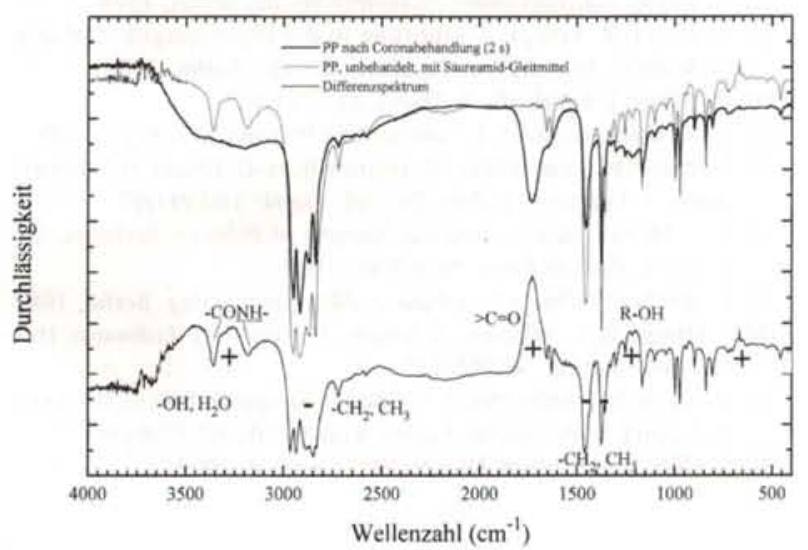

Abb. 12: ATR-Spektren von PP (mit Säureamid-Gleitmittelspuren an der Oberfläche) vor und nach Coronabehandlung, einschl. Differenzspektrum

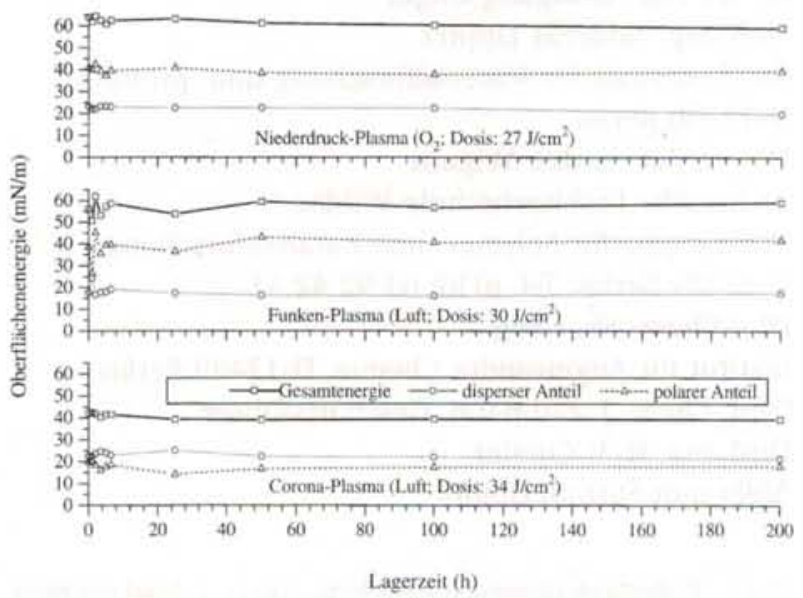

Abb. 13: PP-Oberflächenenergien nach verschiedenen Plasmabehandlungen und ihre Veränderung während der Lagerung
Vergleich der Wirksamkeit der verschiedenen Plasmen In Abbildung 13 sind die Wirksamkeiten der 3 getesteten Plasmavorbehandlungen $\left(\mathrm{O}_{2}\right.$-Niederdruckplasma, Normaldruck-Funkenplasmastrahl mit Luft/ $\mathrm{O}_{2}$ und Coronaentladung) auf die Oberflächenenergie von PP (BOPPFolien) und die Klebfestigkeit zu Polyurethan (PP-Spritzgußmaterial) (Abb. 14) dargestellt (s. jeweils Zeit O s). Die Coronaentladungsbehandlung erreicht etwa Oberflächenspannungswerte von $42 \mathrm{mN} / \mathrm{m}$, der NormaldruckFunkenplasmastrahl von ca. $59 \mathrm{mN} / \mathrm{m}$ und die $\mathrm{O}_{2}$-Glimmentladung von ca. $64 \mathrm{mN} / \mathrm{m}$. Während die Coronabehandlung genügt, um Bedruckbarkeit zu gewährleisten, reicht ihre Wirkung nicht fuir die Herstellung hochhaftfester und alterungsbeständiger Verklebungen aus. Der Funkenplasmastrahl (3D-Treater, Fa. Ahlbrandt) und die Niederdruck-Plasmabehandlung erhöhen die Oberflächenenergie und die Klebfestigkeit besonders stark.

Die relative Wirksamkeit der O-funktionellen Gruppen für die Haftvermittlung hängt stark von dem Plasmaverfahren ab, wie Abb. 15 zeigt. Die höchste Wirksamkeit bezogen auf den gleichen O-Gehalt (XPS-Messungen) besitzt das $\mathrm{O}_{2}$-Niederdruckplasma. Die Interpretation dieser Erscheinung knüpft an die Ergebnisse der NEXAFS-Messungen an, wonach bei der $\mathrm{O}_{2}$-Niederdruckplasmabehandlung ein schmales Zeitfenster existiert, worin schon eine genügende Anzahl polarer Gruppen erzeugt wurde, aber diePolymerstruktur noch in wesentlichen Zügen erhalten geblieben ist. Ähnlich wirkt das

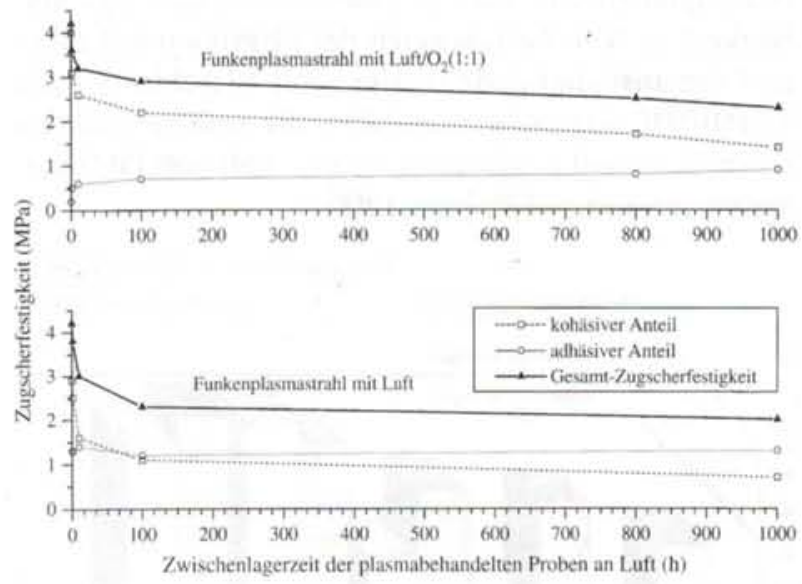

Abb. 14: PP-PU-Zugscherenfestigkeit nach Niederdruck- und Funkenplasmastrahl-Behandlungen und ihre Veränderung während der Lagerung

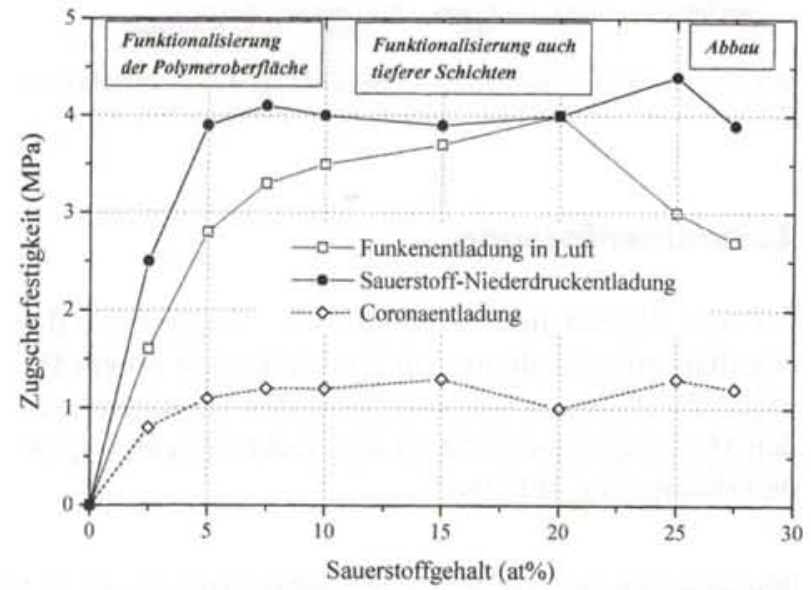

Abb. 15: PP-PU-Zugscherenfestigkeit in Abhängkeit vom Oberflächen-OGehalt (XPS) 
Funkenplasma, während die Coronaentladung offensichtlich die an der Oberfläche liegenden Polymersegmente fragmentierend oxidiert (s. Abb. 12, Zunahme der $\mathrm{C}=\mathrm{O}$-Bande und Abnahme der $\mathrm{CH}_{\mathrm{x}}$-Banden). Der Vergleich zwischen den erzielten Veränderung bei der Oberflächenenergie und in der Klebfestigkeit zu Polyurethan in Abb. 15 für die verschiedenen Plasmavorbehandlungen bestätigt die besondere Wirksamkeit der Niederdruckplasma- und der Funkenplasmastrahlbehandlung.

\section{Stabilität des plasmabehandelten Zustands}

Die Oberflächenenergie einer coronabehandelten additivfreien PP-Oberfläche sinkt während einer $600 \mathrm{~h}$-Lagerung an Luft von $42 \mathrm{mN} / \mathrm{m}$ auf $39,3 \mathrm{mN} / \mathrm{m}$ (s. Abb. 13). Die durch die anderen hier eingesetzten Plasmaarten funktionalisierten Oberflächen unterliegen einer ähnlich geringen Veränderung der Oberflächenenergien während der Lagerung (Funkenplasma: nach 200 h Lagerung: - $1 \mathrm{mN} / \mathrm{m}, \mathrm{O}_{2}$-Niederdruckentladung: auch 200 h Lagerung: $-5 \mathrm{mN} / \mathrm{m}$ ). Deutlicher wird dieser „Fading“-Effekt bei der Betrachtung der Haftung im PU-PP-Verbund (Abb. 14). Für beide Plasmabehandlungen ist ein deutlicher Abfall in der Zugscherfestigkeit zu erkennen, wenn nicht unmittelbar nach der Plasmabehandlung verklebt wurde. Verklebungen, die erst $1000 \mathrm{~h}$ nach der Plasmaaktivierung vorgenommen wurden, zeigten nicht nur eine geringere Haftfestigkeit, sondern trennten sich beim Scheren zu einem hohen Prozentsatz direkt an der Phasengrenzfläche, was für eine alterungsanfällige Verbindung spricht. Extraktionen der PP-Proben mit Ether und die anschließende Analyse des Extrahierten mit MALDI-TOF zeigten beispielsweise die Anwesenheit von Erucasäureamid (Gleitmittel, $\mathrm{m} / \mathrm{z}=304$ ) und PP-Oligomeren von $\mathrm{m} / \mathrm{z}=685$ bzw. 1200 .

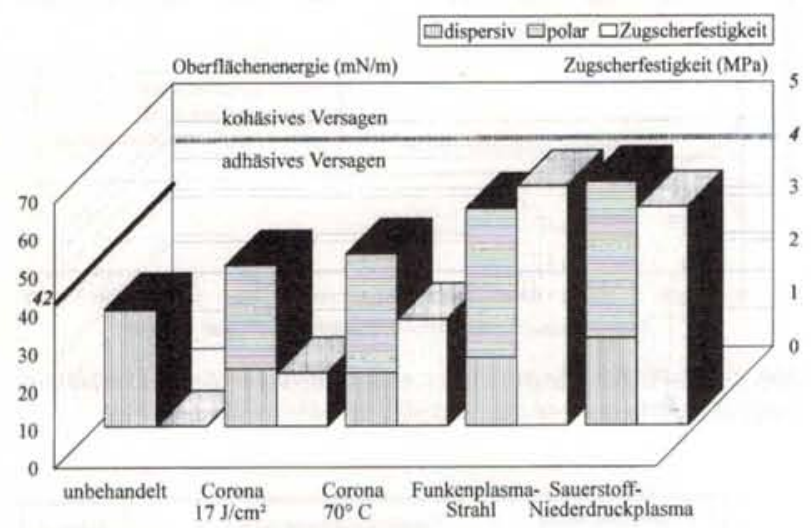

Abb. 16: Vergleich von Oberflächenenergien und Zugscherenfestigkeiten von PP-PU-Verklebungen nach Vorbehandlung in verschiedenen Plasmen

\section{Zusammenfassung}

Für den allgemeinen Zeitablauf der Prozesse bei der Oberflächenbehandlung von Polyolefinen in einem $\mathrm{O}_{2}$ Niederdruckplasma lassen sich aus den Ergebnissen zu den Modellsystemen und zu den Verklebungen folgende Erkenntnisse ableiten:

\begin{tabular}{|c|c|}
\hline Zeitskala & Prozesse an Polymeroberfläche \\
\hline 0 - ca. $2 \mathrm{~s}$ & - Entfernung von Adsorbatschichten \\
\hline $\begin{array}{l}\text { für Vorbehand- } \\
\text { lungen zu } \\
\text { bevorzugendes }\end{array}$ & $\begin{array}{l}\text { Konformations- und Konfigurationswechsel von } \\
\text { einzelnen Gruppen und Kettensegmenten ver- } \\
\text { ändern die Oberflächenenergie (dispersiv) }\end{array}$ \\
\hline Zeitsegment & - Bildung von Radikalen \\
\hline ab ca. $0.1-2 \mathrm{~s}$ & $\begin{array}{l}\text { - Beginn der plasmagasspezifischen Funktionali- } \\
\text { sierung der Makromoleküle an der Polymerober- } \\
\text { fläche (1,5-25 at.\%) }\end{array}$ \\
\hline ab ca. $2 \mathrm{~s}$ & 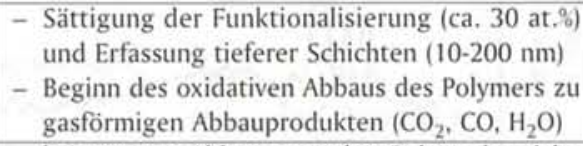 \\
\hline bis ca. $300 \mathrm{~s}$ & $\begin{array}{l}\text { - bevorzugter Abbau amorpher Polymerbereiche } \\
\text { und amorpher Ränder von Polymerkristallen (se- } \\
\text { lektives Ätzen) } \\
\text { - Bildung olefinischer/konjugierter Doppelbin- } \\
\text { dungen in der Oberflächenschicht und z. T. im } \\
\text { Polymervolumen (bis einige } 10 \mu \mathrm{m} \text { tief) }\end{array}$ \\
\hline bis ca. $3600 \mathrm{~s}$ & $\begin{array}{l}\text { - Einstellung eines stationären Zustandes zwi- } \\
\text { schen Funktionalisierung, Bildung von Doppel- } \\
\text { bindungen, örtlichen Vernetzungen sowie Ket- } \\
\text { ten und Kristallabbau }\end{array}$ \\
\hline Post-Plasma - & $\begin{array}{l}\text { - kein meßbarer Einbau von Luftsauerstoff } \\
\text { (additivfreie Oberfläche) }\end{array}$ \\
\hline
\end{tabular}

Sinngemäß und mit einer deutlich verkürzten Zeitskala gilt dieses Schema auch für die Funkenplasma- und die Corona-Vorbehandlung.

\section{Literatur}

[1] H. Drost, Plasmachemie, Akademie-Verlag, Berlin, 1978

[2] G. Hertz, R. Rompe, Einführung in die Plasmaphysik und ihre technische Anwendung, Akademie-Verlag, Berlin, 1968

|3| W. Stiller, J. Friedrich, Z. Chem. 21(1981)91

|4] J. Friedrich, G. Kuhn, J. Gähde, Acta Polymerica 30(1979)470

|5| J. Friedrich, I. Loeschcke, H. Frommelt, H.-D. Reiner, H. Zimmermann, P. Lutgen, J. Polym. Degrad. Stabil. 31(1991)97

6] K. L. Mittal, Physicochemical Aspects of Polymer Surfaces, Bd. 1 und 2, Plenum Press, New York, 1983

17] C. Bischof, W. Possart, Adhäsion, Akademie-Verlag, Berlin, 1982

[8] J. Friedrich, L. Wigant, W. Unger, A. Lippitz, J. Erdmann, H.V. Gorsler, Coating, 4(1995) 123

|9| J. Friedrich, J. Erdmann, H.-V. Gorsler, W. Saur, P. Rohrer, W.Unger, A. Lippitz, GAK-Gummi, Fasern, Kunststoffe 47(1994)382

[10] S. Hild, Dissertation, Universität Clausthal, 1994

\section{Verfasser}

Dr. rer. nat. habil. Jörg Friedrich

Dr. rer. nat. Wolfgang Unger

Dipl.-Ing. Andreas Lippitz

Bundesanstalt für Materialforschung und -prüfung,

D-12200 Berlin

Dr. rer. nat. Lothar Wigant

Technische Fachhochschule Wildau

WIP-Gruppe für Polymer- und Katalyseforschung

D-12489 Berlin; Tel. (030) 63924251

Dipl-Chem. Sh. Geng

Institut für Angewandte Chemie, D-12489 Berlin

Dipl.-Chem. J. Erdmann, Geschäftsführer

Dipl.-Ing. H.-V. Gorsler

Ahlbrandt System GmbH 